\title{
Henry E. Sigerist (1891-1957) \\ und die erste medizinhistorische Konferenz in Pura (Tessin)
}

Zur Erinnerung an den 25./26. März 1950*

Von Heinrich Buess

Im Herbst 1979 fand sich bei der Durchsicht meiner privaten Korrespondenz eine Anzahl von Dokumenten, welche die sogenannten Pura-Konferenzen (Sigerist-Tagungen) betreffen. Diese Briefe und Photos legten es nahe, in unserem Gesellschafts-Organ an den Auftakt dieser überaus anregenden Stunden des kollegialen Zusammenseins zu erinnern. Dieser Gedanke drängte sich um so mehr auf, als die meisten Teilnehmer des Pura-Kreises gestorben sind und als sich bei den noch lebenden Kollegen keine persönlichen Erinnerungen an die erste Tagung erhalten zu haben scheinen.

In den von Nora Sigerist Beeson herausgegebenen "Autobiographical writings" (1966) fehlt ein Hinweis aus der Feder Sigerists, was verständlich ist, da der Veranstalter selber schon früher über diese Frühjahrs-Konferenz berichtet hatte, nämlich in seinem ausführlichen "Report" über "The History of Medicine in Switzerland" (1952). Sigerist erinnert in ungezwungener Weise an seine ersten Eindrücke in der Schweiz, wobei er die im Herbst stattfindenden Haupttagungen der Schweizerischen Gesellschaft für Geschichte der Medizin und der Naturwissenschaften mit Recht zuerst nennt.

In der ersten breit angelegten biographischen Würdigung Sigerists aus der Feder von Elisabeth Berg-Schorn $(1978)^{1}$ ist der Eindruck von Prof. Hans Schadewaldt (Düsseldorf) über eine spätere Konferenz zutreffend geschildert. In dem von Sigerists jüngerer Tochter Nora aufbewahrten Nachlaß soll es eine spezielle Mappe mit Dokumenten über die Pura-Konferenzen geben (siehe S.228 unten).

* Frau Erica Campanella-Sigerist (Grand-Saconnex/Genf), Herrn Dr. med. Charles Salzmann (Rüschlikon/Zürich), Herrn Dr. med. Achille Piotti (Lugano) und Herrn Prof. Nikolaus Mani (Bonn) sei für ihre Bemühungen um die Identifikation der Personen auf der Photographie (die von Herrn Prof. Sigerist gemacht wurde) herzlich gedankt.

${ }^{1}$ Den ersten Hinweis auf diese Dissertation verdanke ich Herrn Prof. E. H.Ackerknecht (Zürich); vgl. deren Rezension in Gesnerus 36 (1979), H.3/4, S.315-316. Der Initiantin dieses Lebensbildes, Frau Prof. M.Putscher (Köln), danke ich für das geschenkweise überlassene Dissertations-Exemplar. 
Mir geht es hier vor allem darum, das «Ambiente» dieser für jeden damaligen Teilnehmer unvergeßlichen Tage aufleben zu lassen. Zuvor sei indessen ihre Vorgeschichte mitgeteilt.

\section{I}

Dem an der Persönlichkeit Sigerists interessierten Leser dürften die soeben genannten "Autobiographical writings" des bedeutenden Gelehrten bekannt sein. Sie sind 1966 von der McGill University Press in Montreal herausgegeben worden und gestatten tiefere Einblicke in das Denken und Fühlen ihres Verfassers. Die Kleinigkeiten des Alltags sind, abgesehen von den eigenen Kochkünsten und anderen Details, kaum erwähnt. Die große Politik und die Wandlungen im Gesundheitswesen erlebt der Leser wie durch ein Vergrößerungsglas, in dem sich die Ereignisse durch das «Mitleiden» Sigerists in ihrer ganzen Dramatik abzeichnen. Aktivitäten in der Medizingeschichte kommen vor allem hinsichtlich seiner eigenen Gastvorlesungen zur Sprache. Unter den beiden Vorkommnissen, in denen es ihm an Mut gefehlt habe, nennt unser Mentor am 28. Mai 1946 im Rückblick seine Entscheidung, «als ich die Leitung der Welch Library annahm». Der Mangel an helfenden Kräften, die ihm aus finanziellen Gründen verweigert wurden, habe seine Gesundheit und sein Lebenswerk zerstört.

Diese Einsicht führte Sigerist im Herbst 1946 zu dem Entschluß, die ihm noch verbleibenden Lebensjahre der wissenschaftlichen Forschung in einem abseits gelegenen stillen Ort in seiner Heimat zu widmen. Die Liebe zu Italien, zu dem Land, «wo die Citronen blühn», zu einem «alten Haus mit großen Räumen und hohen Decken» (S.202) bestimmte seine Wahl. Und an Ostern (6. April) 1947 meldet er tief beglückt, daß die «Casa Serena» in Pura (Tessin) von seinen Angehörigen in der Schweiz für ihn gefunden worden sei. "56 years old! The fortress is battered but still holding. At the end of the seminar the students presented me with a bottle of rare cognac, milk for an old man." (7. April). - In Basel angekommen, freut er sich über die «Alternative» zum bisherigen Leben eines Universitätsdozenten, mit andern Worten: «seinen Unterricht durch seine Bücher» zu ersetzen. Am 19. Juli erfolgt der Einzug in das «charmante Haus» mit seiner unvergleichlichen Aussicht.

Was den sogenannten «Sigerist-Konferenzen» in der Schweiz unmittelbar voranging, läßt sich auf Grund der von mir aufbewahrten Briefe miterleben, wobei wir uns allerdings auf eine kleine Auswahl von Stellen beschränken müssen. 
«In den letzten Tagen wurde zwischen Herrn Dr. Milt, Herrn Dr. Wehrli und mir eine Zusammenkunft in Aarau vereinbart, auf der die Medizinhistoriker der Schweiz in gemütlicher Weise einige Stunden beieinander sein und ihre Gedanken austauschen können. Es handelt sich bei diesem Treffen um eine völlig 'inoffizielle' Zusammenkunft, die dazu dienen soll, den Kontakt zwischen den Männern unseres Faches etwas intensiver zu gestalten.» Diese Einladung (9.Oktober 1948) wurde vom Verfasser dieses Berichtes an seinen Lehrer, Herrn Dr. med. Johannes Karcher (1872-1958), der seit 1939 an der Universität Basel Ehrendozent für Geschichte der Medizin war, gerichtet. Karcher, ein überaus kultivierter Internist, der als Mitglied der Curatel im Präsidium unserer Hochschule lange Jahre einen großen Einfluß ausgeübt hatte, konnte aus gesundheitlichen Gründen nicht zum «Hock» nach Aarau kommen (Brief vom 18. Oktober 1948). Er sollte 1 1/2 Jahre später in Pura mit besonderer Verehrung bedacht werden.

Die eben zitierte Stelle aus der Einleitung unseres Briefes wurde ausgewählt, um anzudeuten, daß die in Pura seit 1950 alljährlich abgehaltenen Konferenzen eine kurze Vorgeschichte haben. Nach meiner Habilitation (1946) und der Teilnahme an den offiziellen Kongressen im Rahmen der Schweizerischen Naturforschenden Gesellschaft enttäuschte mich vor allem das Fehlen eines persönlichen Gedankenaustausches unter den Schweizer Fachleuten. Der erste Kollege, dem dieses Gefühl zum Ausdruck gebracht wurde, war der Zürcher Internist und spätere erste Professor für unser Fachgebiet, Bernhard Milt (1896-1956). In überaus verständnisvoller Weise ging Milt auf mein Anliegen ein, und am 31. Oktober 1948 fand im «Aarauer Hof » in Aarau ein informelles Treffen statt, an dem auch Prof. Sigerist teilnahm. Wie die übrigen Fachvertreter schätzte er, wie er mir schrieb, besonders die kleine Zahl der an dem Anlaß beteiligten Ärzte.

Während des Jahres 1949 trafen wir uns im Frühling und im Herbst in Aarau bzw. in Olten in ebenso zwangloser Weise, wobei auch der als Sammler von Wachsplastiken bekannte Prof. Edgar Goldschmid (1881-1957) aus Lausanne mit von der Partie war. Auf die Durchführung der Sitzungen sei hier nicht eingegangen. Irgendwelche weiterreichenden Beschlüsse wurden nie gefaßt. Hingegen blieb bei mir das «Malaise» um die offiziellen Kongresse bestehen, und in einem Brief an Prof. Sigerist (6. September 1949) machte ich meinem Ärger Luft über die «unglückliche Organisation» von seiten des Vorstandes. Der Empfänger des Schreibens teilte mein «Urteil über die Tagung in Lausanne... vollkommen», «denn es ist ganz klar, daß es in dieser Weise nicht weitergehen kann, wenn die Gesellschaft nicht steril werden soll» (Brief vom 17. September 1949). 
Sigerist nahm sich eine Diskussion der Frage am Aarauer Treffen vom Oktober 1949 vor. Doch dürfte in ihm schon damals der Gedanke aufgetaucht sein, die Schweizer Historiker der Medizin und der Naturwissenschaften nach Pura einzuladen. Inzwischen war nach dem Tod von PD Dr. Gustav Adolf Wehrli (18881949), über den Urs Boschung im vorliegenden Heft des Gesnerus berichtet, auch die Habilitation von Bernhard Milt erfolgt. Am 11.September 1949 schrieb er mir, daß in Pura eine neue Pension eröffnet worden sei und daß am «letzten Aprilsonntag eine zweitägige Tagung unseres Aarauerkreises vorgesehen» sei. Die Anregung sei von Prof. Sigerist ausgegangen. Es ist verständlich, daß dieses unerwartete Anerbieten aus Pura uns freudig überraschte und immer mehr anzog.

Was wir uns von dieser Tagung erhofften, waren ein intensiverer Kontakt und eine verbesserte Vortragsmöglichkeit sowie eine umfassende Orientierung über alles, was in unserm Fachgebiet vor sich ging. Der von Sigerist schon in Aarau vermittelte «Tour d'horizon» sollte auch in Pura ein regelmäßiger Programmpunkt sein, wobei das Schwergewicht durchaus auf der Geschichte der Medizin liegen würde.

\section{III}

Ein wesentliches Anliegen war Prof. Sigerist und dem Schreibenden die Förderung des wissenschaftlichen Nachwuchses. Bei seiner Vorbereitung der Tagung in Pura kam diese Zielsetzung in mehreren Briefen zum Ausdruck. Auch bei den späteren Einladungen wurden die jüngeren Kollegen aus der Schweiz und aus Deutschland (BRD) immer mehr berücksichtigt. Die heute aktive Generation der Medizinhistoriker war je länger je mehr vertreten. In dem erfahrenen Gelehrten, der die Verhältnisse in der Alten und in der Neuen Welt kannte, besaßen wir alle einen bewährten Mentor. So war von der ersten Konferenz an in der Basler Delegation der junge Bündner Arzt Nikolaus Mani, auf dessen Mitarbeit unser Gastgeber großes Gewicht legte, mit von der Partie.

Im Besitz des Schreibenden findet sich eine sozusagen vollständige Dokumentation über sämtliche Konferenzen von Pura «in Wort und Bild». Hingegen sei an dieser Stelle aus Platzgründen nur der Anlaß von 1950 vor Augen geführt.

Am 8. März 1950 ging aus der "Casa Serena» in Pura die Einladung an die Gäste ab. Die erste Abbildung, die das Programm gewissermaßen als Vorspann zierte, zeigt das gastliche Heim des Ehepaares Sigerist, aufgenommen wohl durch den Initiator dieser «Konferenz» vom 25./26. März 1950.

Das Programm wird hier im Wortlaut wiedergegeben: 


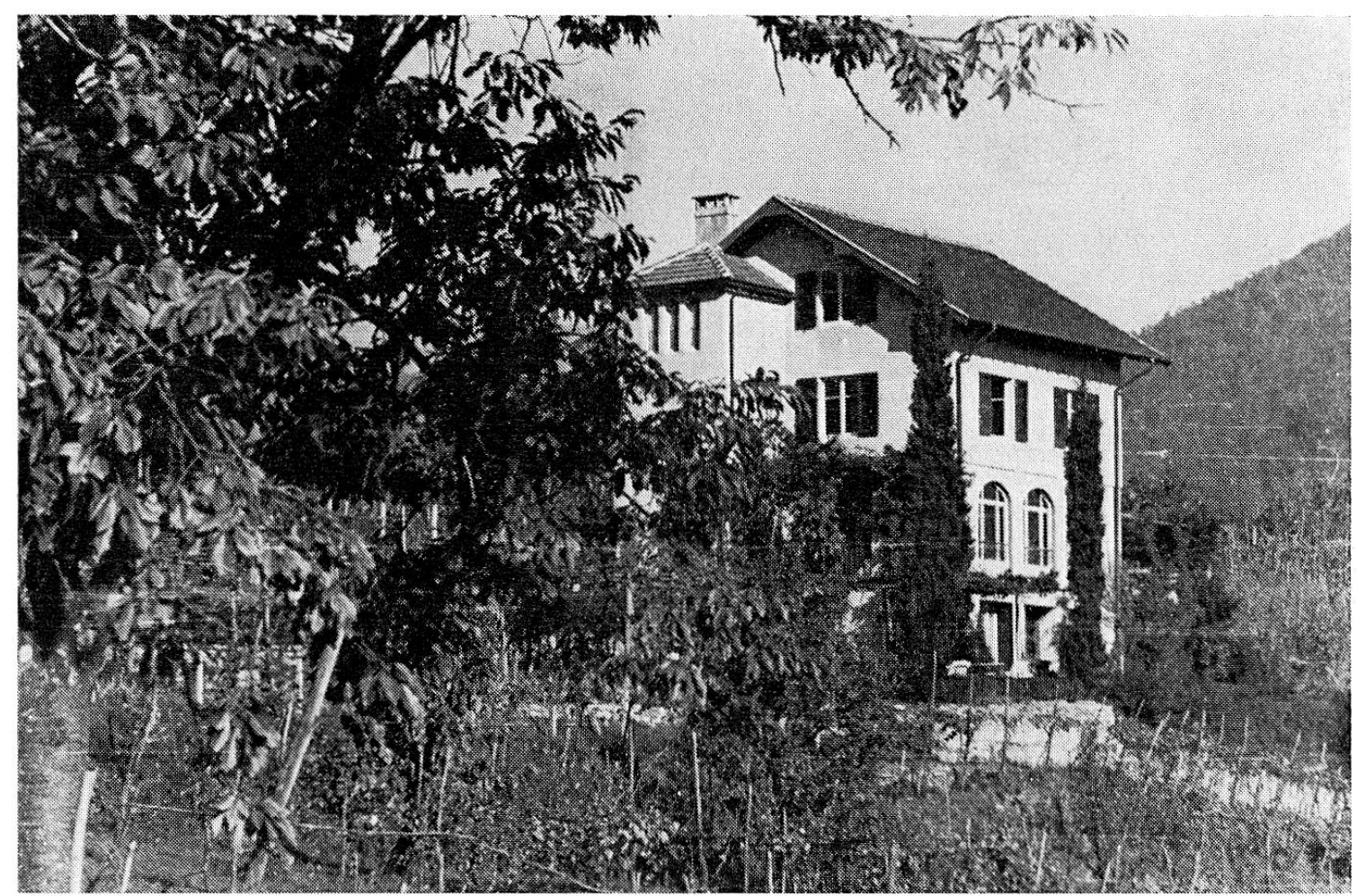

Abb.1. Casa Serena, das Haus Henry E.Sigerists in Pura

Samstag, den 25. März

Ankunft in Lugano

Von Zürich: 13.23.

Von Basel-Bern: 14.19.

Die Teilnehmer werden am Bahnhof Lugano abgeholt und im Auto des Albergo Paladina nach Pura gefahren.

\subsection{I.Sitzung: In Casa Serena}

1) Ch.Salzmann (Zürich): Medizinalgeschichte des Tessin.

2) E.Hintzsche (Bern): Bibliothek eines Schweizer Arztes des 15. Jahrhunderts.

3) H. Buess (Basel): Streiflichter auf das helvetische Militärsanitätswesen.

19.00 Abendessen im Albergo Paladina

\subsection{Sitzung: In Casa Serena}

1) Geschäftliches und Tour d'Horizon.

2) H.E. Sigerist (Pura): Anfänge der griechischen Heilkunde.

3) Geselliger Teil. 


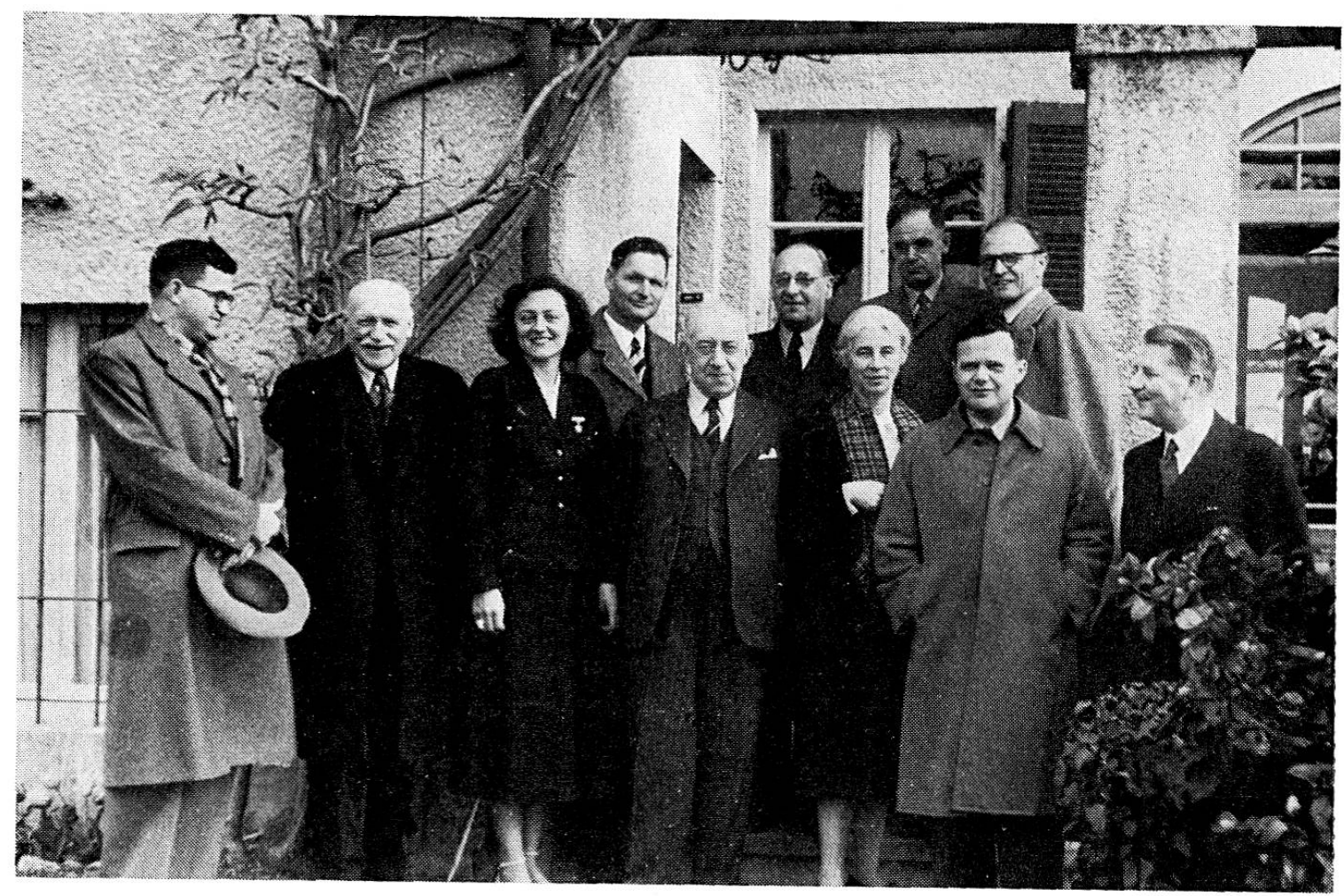

Abb.2. Vordere Reihe von rechts nach links: Charles Salzmann, Nikolaus Mani, Frau Emmy Sigerist-Escher, Edgar Goldschmid, Sekretärin von H.E.Sigerist, Johannes Karcher, Heinrich Buess

Hintere Reihe von rechts nach links: Erich Hintzsche, Bernhard Milt, Karl Reucker, Eduard Fueter

Sonntag, den 26. März

\subsection{III.Sitzung: In Casa Serena}

1) N.Mani (Andeer-Basel): Aus der Geschichte der Leberphysiologie.

2) E. Goldschmid (Lausanne): Über die beiden Bell.

3) E. Fueter (Wädenswil): Arnold J.Toynbees Werk "A Study of History" und die Geschichte der Wissenschaften.

13.00 Mittagessen im Albergo Paladina

Spaziergang zur Rochuskapelle auf dem Weg zwischen Pura und Curio.

Ab Lugano

Nach Zürich : 17.12 .

Nach Basel-Bern: 17.54. 
Auch die im Programm erwähnte Rochus-Kapelle «zwischen Pura und Curio 1576-1857» sowie das reizende Dörfchen Pura waren in teils selbst verfertigten Photos der Einladung beigelegt. Der in den Pausen mit Kaffee aufwartende Gastgeber, in dessen großer Halle die Sitzungen stattfanden, ließ es sich ferner angelegen sein, jedem der Teilnehmer später ein von ihm geknipstes Gruppenbild zu schicken (Abb.2).

In besonderer Weise trugen zur Verschönerung der Tagungen die Reize des ersten Frühlingszaubers bei. Meine Eindrücke versuchte ich in einer Zeitungsnotiz zu schildern (auszugsweise mitgeteilt aus Nr.157, 14. April 1950 der «Basler Nachrichten »):

«Wer sich, sei es mit der Bahn oder auf den von blühenden Sträuchern umsäumten Wanderwegen, entlang dem reizvollen Agneser Zipfel des Luganersees nach dem idyllisch gelegenen Ponte Tresa begibt, wird in diesen Tagen, wo die Rebberge noch kahl dastehen, auf einem in die 'Campagna' von Magliaso vorspringenden Hügel ein hochgebautes Haus erblicken, das den früheren Besuchern des Dörfchens Pura vielleicht noch als 'Grotto' in Erinnerung ist. Ein einzigartiger Blick bietet sich dem Besucher dieses Hauses, wenn er durch die von Sonnenwärme durchflutete Pergola auf die schmale Treppe zugeht, die an der Seitenwand emporklettert...

Tritt nun der Besucher über die Schwelle des hochragenden Hauses, so empfängt ihn dort eine andere Welt, nicht minder weit und vielgestaltig, die Welt der Bücher. Nicht mehr leiblichen Genüssen, sondern ernstem wissenschaftlichem Arbeiten dienen die im Dämmerlicht daliegenden innern Räume, deren Wände von oben bis unten mit Bücherregalen ausgekleidet sind. Denn hier hat ein Gelehrter von Weltruf seinen Wohnsitz aufgeschlagen, unser Landsmann Henry E. Sigerist, der vor einigen Jahren die Hetze der Großstadt mit der stillen Klause des Malcantone vertauscht hat. Wenn Männer um das 60. Altersjahr sich vom aktiven Leben zurückziehen, so nimmt man an, daß sie sich dem «otium cum dignitate» verschrieben haben. Nicht so unser Kollege, dessen Mutter übrigens hochbetagt in unserer Stadt lebt. Nach einer sich fast über ein Vierteljahrhundert erstreckenden Lehrtätigkeit in Zürich, Leipzig und an der John Hopkins Universität in Baltimore, wo er der Medizingeschichte der Neuen Welt entscheidende Impulse verliehen hat, ließ sich Prof. Sigerist in Pura nieder, um an einem großen medizingeschichtlichen Werk zu arbeiten, dessen erster Band sich bereits im Druck befindet.

Äußerst wertvoll war auch jetzt wieder der an unseren Tagungen bereits fest eingebürgerte «Tour d'horizon» des Gastgebers, in dem über den Stand der medizingeschichtlichen Forschung und des Unterrichts, der ja in vielen Ländern weit besser entwickelt ist als in der Schweiz, die neuesten Mitteilungen gemacht wurden. Als besonders anregend erwies sich das gesellige Beisammensein im neu hergerichteten 'Albergo Paladina', wobei namentlich im Gespräch mit den älteren Teilnehmern, unter ihnen auch Dr. J. Karcher und Dr. K. Reucker aus Basel, persönliche Erfahrungen ausgetauscht wurden. In angeregter Diskussion, die bis über die mitternächtliche Stunde hinaus fortgesetzt wurde, ergaben sich da und dort neue Gesichtspunkte. Die Atmosphäre des Hauses und die Anwesenheit von Gelehrten mit den verschiedensten Interessen, unter denen noch 
der Zürcher Fachvertreter Bernhard Milt zu nennen ist, verlieh der Tagung nicht nur ein hohes wissenschaftliches Niveau, sondern auch einen besonders intimen Charakter. Und es hat den Anschein, als ob sich Pura zu einem Zentrum der Pflege der Wissenschaftsgeschichte und der Kulturgeschichte im weiteren Sinn entwickeln würde, denn im Spätsommer dieses Jahres sollen sich im schönen Heim von Prof. Sigerist eine Anzahl Gelehrte aus dem angelsächsischen Sprachgebiet versammeln, um geschichtliche Fragen der 'sozialen Medizin' zu studieren. Auch wir in Basel freuen uns darüber, daß an den Abhängen des schönen und uns so vertrauten Luganersees - vergleichbar den 'Eranos'Tagungen am 'Lago Maggiore' - ein fruchtbarer Mittelpunkt wissenschaftlicher Gespräche emporzublühen im Begriffe ist.»

\section{Schlu $\beta$}

In der Weihnachtswoche 1950 hatte Sigerist geschrieben: "We also know that death may come unexpectedly from an unrelated accident or some intercurrent disease that a weakened heart is unable to overcome. But whatever fate may have in store for us, we must be prepared to accept it and to die as we have lived." (Sigerist, 1952.)

An diese Sätze denkt man in der Erinnerung an den Tod Henry E. Sigerists am 17. März 1957. Ein Jahr vorher (18. März 1956) war der Inhaber des außerordentlichen Lehrstuhls in Zürich, Bernhard Milt, ganz plötzlich gestorben, und im Einverständnis mit dem Veranstalter wurde für 1956 auf eine Tagung verzichtet. Auf die vielleicht im Zusammenhang mit den Pura-Tagungen letzte Äußerung eines persönlichen Wunsches des vom Tode gezeichneten Gelehrten sollte, auch im Sinne seiner Gattin, die 8. Medizinhistorische Konferenz am 30. und 31.März 1957 durchgeführt werden (Einladung von Dr. Salzmann, datiert aus Pura von 19. März 1957).

So haben während zehn Jahren (1948-1957) diese privaten Treffen von schweizerischen und einzelnen ausländischen Medizinhistorikern bestanden. Dieser Seitensproß wissenschaftlichen und geselligen Zusammenseins war in der milden Sonne des Tessins zu schönster Blüte gediehen. Die kosmopolitische Weite des Denkens und Fühlens von Henry E. Sigerist hatte eine der historischen Diskussion günstige Atmosphäre geschaffen. Nach seinem Hinschied fehlte der begeisternde «Spiritus rector». Ein verheißungsvolles Intermezzo war zu Ende gegangen. 


\section{Literatur}

Berg-Schorn, Elisabeth, Henry E. Sigerist (1891-1957), Medizinhistoriker in Leipzig und Baltimore, Standpunkt und Wirkung, Diss. med. Köln 1979 (Buchhandelsausgabe: Köln 1978).

Sigerist, Henry E.

a) Living under the shadow. The Atlantic Monthly 189 (1952), S.25-30.

b) The History of Medicine in Switzerland. Bull. History Med. XXVI (1952), S.187-190.

c) Autobiographical writings. Selected and translated by Nora Sigerist Beeson. Montreal (McGill University Press) 1966.

Vescia, Fernando G., Henry E.Sigerist: The years in America. Medizinhistorisches Journal 14 (1979), S.218-232.

\section{Summary}

This short article is a souvenir of the so-called "Pura-Konferenzen", at least of the first one (25/26 March 1950). Using his private correspondence, the author shows the beginnings of these meetings of the Swiss representatives of Medical History with Prof. Sigerist in Aarau and Olten. In Pura they met during eight years, until Spring 1957 (after the death of Sigerist). The first program and a photo of the participants may be of special interest.

Prof. Dr. med. Heinrich Buess

Medizinhistorisches Institut

Klingelbergstraße 23

CH-4031 Basel 